\title{
Ab Initio Many Electron Relativistic Molecular DFS-Calculations of Inner Shell MO-Transitions ${ }^{+}$
}

\author{
B. Fricke, W.-D. Sepp, and T. Morović \\ Fachbereich 18-Physik, Universität Kassel, Postfach 101380 , \\ D-3500 Kassel, Federal Republic of Germany
}

Received August 2, 1984

\begin{abstract}
Using a relativistic molecular DFS code we have calculated a self-consistent many electron relativistic correlation diagram of the system Cl-Ar. The resulting energy for the $2 \mathrm{p} \pi-1 \mathrm{so}$ transition is in complete agreement with the experimental results of Schuch et al./1/. This definitely confirms their claim that they are able to measure this quantity from interference structures observed in quasi-molecular $\mathrm{K}-\mathrm{X}-\mathrm{ray}$ spectra.
\end{abstract}

Since MO-X-rays became an interesting part of heavy ion atom collision physics many groups have tried to determine the molecular orbital binding energies as function of internuclear distance, and thus to measure correlation diagrams. Up to now nobody was really able to gain this interesting information. The deternination of the end-point in the MO-X-ray spectra failed because of the collision broadening, and the maxima in the anisotropy of MO-X-ray emission could not be correlated unequivocally to Mo transition energies. This whole field was reviewed in several articles over the years $/ 2,3 /$. The most recent article, with all important references, is in preparation by Anholt/4/.

Recently schuch et al./1/ published a paper in which they report their observations of interference structures of quasimolecular $\mathrm{K}-\mathrm{X}$-rays at various impact energies of highly stripped $\mathrm{Cl}^{16+}$ on $\mathrm{Ar}$ at fixed impact parameters. Using the stationary phase approximation/5/ for the quasi-molecular dipole transition $/ 6 /$, they are able to obtain information on the phase factors which consist mainly of the time integral of the aifference of the energy eigenvalues of the Mo transitions during collision.

As the system $\mathrm{Cl}^{16+-A r}$ is very well defined with one incoming electron in the is level of the lighter partner only, the observed interference structure is interpreted by the authors/1/ as a determina- tion of the $2 \mathrm{p} \pi-1 \mathrm{so}$ transition energy in this quasi-molecule. Their experimental results are shown in Fig.1. They compare these values with MO-binding energies which were obtained by scaling from the quasi-molecular energies of the $\mathrm{H}^{+-} \mathrm{H}$ system, according to the procedure given in ref.7. The dashed line represents the $2 \mathrm{p} \pi-1 \mathrm{~s} \sigma$, and the dot-dashed one the $2 \mathrm{p} \sigma-$ 1so transition energy. From general arguments concerning dipole transition matrix elements and population of these states, they conclude that their experimental points represent the $2 \mathrm{p} \pi-1 \mathrm{~s} \sigma$ transition, although the scaled "theoretical" curve (dotted line) is systematically higher. If this interpretation is correct, this measurement would be the first direct

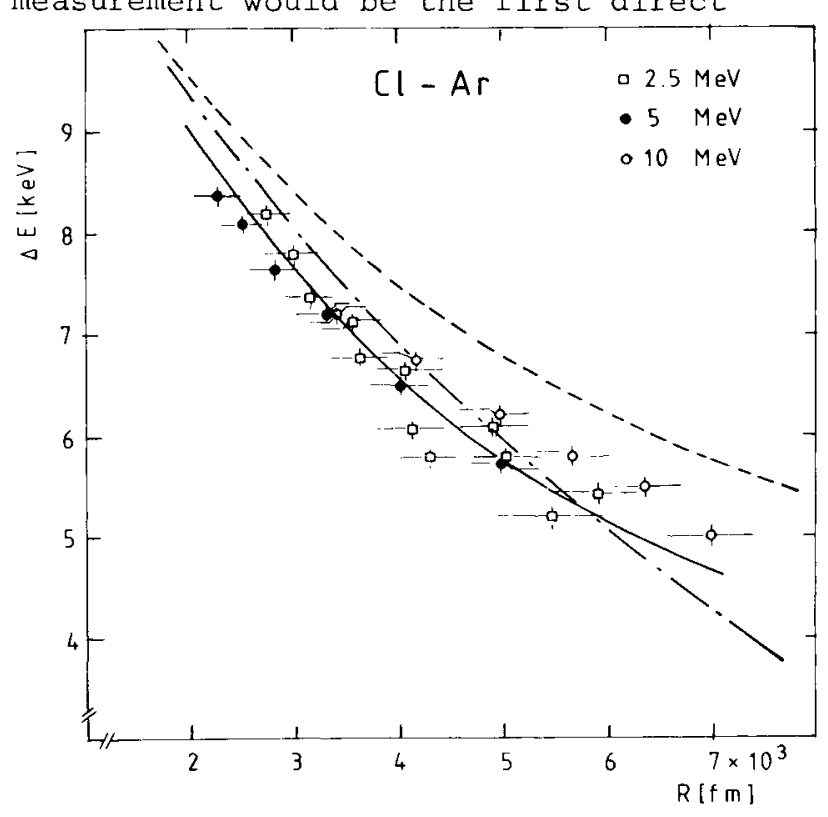

Fig.1: Quasi-molecular 2pm-1so transition energy as function of internuclear distance. Experimental data are from Schuch et al./1/. Dashed curve (dot-dashed curve) $2 \mathrm{p} \pi-1 \mathrm{~s} \sigma(2 \mathrm{po}-1 \mathrm{so})$ transition energy acc. to ref.1. Full curve $2 \mathrm{p} \pi-1 \mathrm{~s} \sigma$ transition energy from our DFS calculations. 
experimental determination of the difference of two electronic states in a quasimolecule as function of internuclear distance. To confirm or disapprove this supposition it is, therefore, important and neccessary to compare with much more accurate theoretical transition energies.

For this reason we have calculated the Cl-Ar correlation diagram with 10 electrons removed, using our ab initio molecular relativistic DFS code/8/ were the interaction of all electrons in this system as well as relativity are included from first principles. We obtain a correlation diagram which is presented in Fig. 2. As this calculation is done fully relativistic we use the nomenclature which counts the levels according to their projection of the total angular momentum on the internuclear axis. The $1(1 / 2)$ level is identical with the 1 so level in the usual non-relativistic nomenclature, the $2(1 / 2)$ level is the $2 \mathrm{po}$ level, and the $2 \mathrm{p} \pi$ level is relativistically split into the nearly degenerate $1(3 / 2)$ and $3(1 / 2)$ levels at internuclear distances $R>0.03$ a.u. The $2 \mathrm{p} \pi$ level has a relatively constant energy eigenvalue up to internuclear distances of $\mathrm{R} \approx 0.1 \mathrm{a} . \mathrm{u}$. , which can be seen in many medium $z$ electron correlation diagrams. If we use the difference of the energy eigenvalues of the $2 \mathrm{p} \pi$ and $1 \mathrm{~s} \sigma$ level in $\mathrm{Fig.2}$, we obtain the values which are represented by the full curve in Fig. 1.

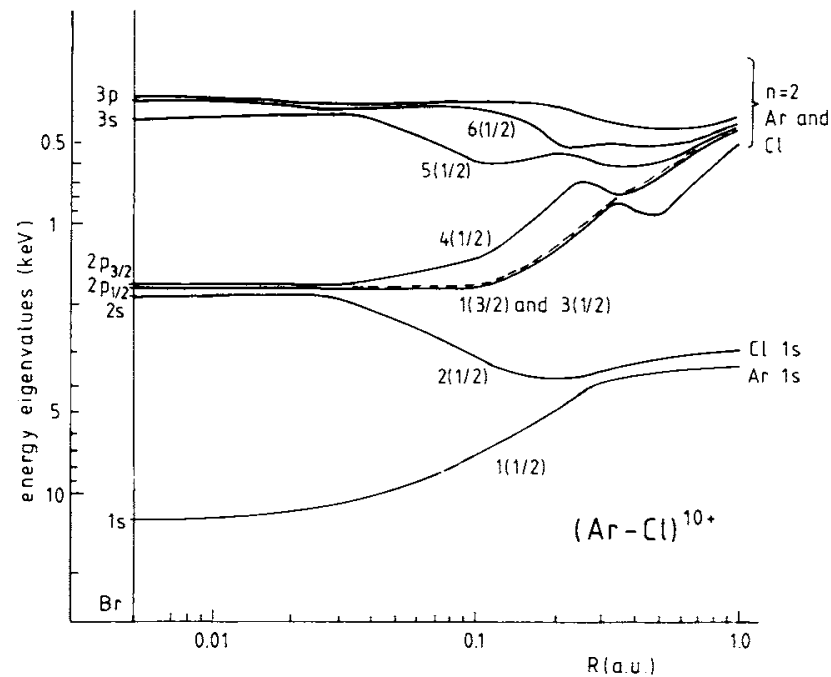

Fig.2: SCF relativistic many electron correlation diagram of (Ar-Cl) $10+$.
This curve is in beautiful agreement with the experimentally determined transition energies of Schuch et al./1/. As this correlation diagram is the best one can calculate at present, and it includes screening and relativity, this result definitely confirms that they really found a method which is able to determine the $2 \mathrm{p} \pi-1 \mathrm{~s} \sigma$ inner shell Mo-transition energy. One reason that the experimental curve has a relatively large energy spread may very well come from different occupations of the innermost shells which then shift the transition energy up to a few hundred ev.

In conclusion one may say that the good agreement between experiment and theory shows that the method developed by schuch et a1./1/ indeed opens the way for spectroscopic studies of molecular orbitals. It is hoped in particular, that it actually can be a potential tool for the study of superheavy quasi-molecules, were such experimental results could lead to a testing method of screening, relativistic, and QED effects. The latter are of spe-

cial interest in this region of elements with $\mathrm{z} \alpha>1$ from a general point of view.

+ Supported by Gesellschaft für Schwerionenforschung, Darmstadt.

\section{References}

1. R. Schuch, H. Schmidt-Böcking, I. Tserruya, B.M. Johnson, K.W. Jones, and M. Meron; Phys.Rev. Lett., in print.

2. W. Meyerhof; Science 3 , 839 (1976).

3. W. Lichten; J.Phys.Chem, 84, 2102 (1980).

4. R. Anholt; Rev.Mod.Phys., in print.

5. J.N.L. Connors; J.Chem.Phys. 55, 5636 (1971).

6. J. Macek and J.S. Briggs; J.Phys. B7, $1312(1974)$.

7. K.H. Heinig, H.U. Jäger, H. Richter, H. Wättenek, W. Frank, P. Gippner, K.H. Kaun, and P. Manfrass; J.Phys. B10, 1321 (1977) .

8. W.-D. Sepp, W. Sengler, D. Kolb, H. Hartung, and B. Fricke; Chem.Phys. Lett., in print and, to be published. 